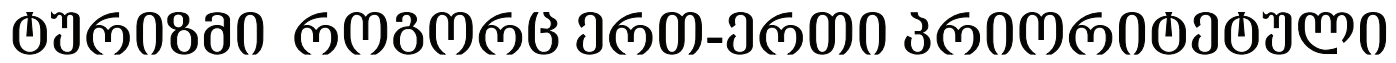

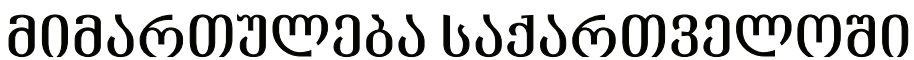

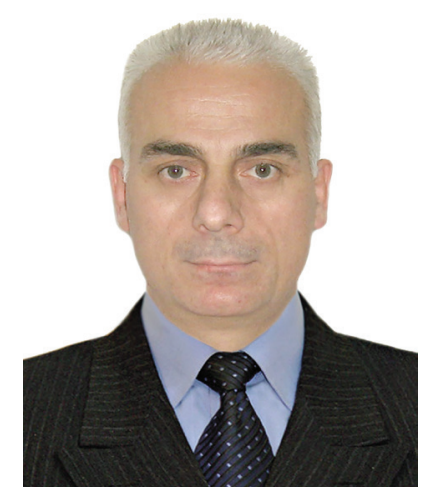

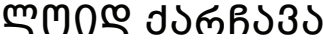

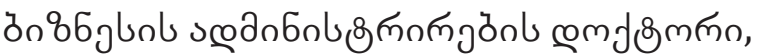
उnmogulumn.

jm.oुmbş:loidk@yahoo.com

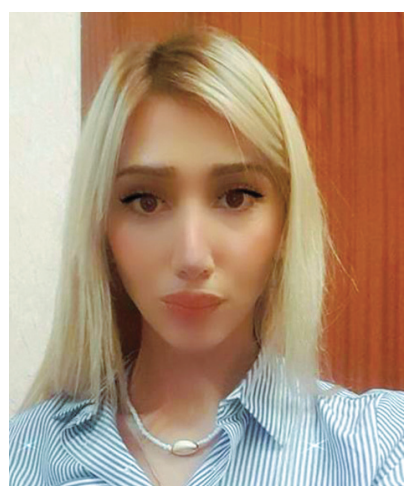

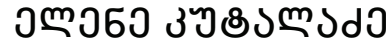

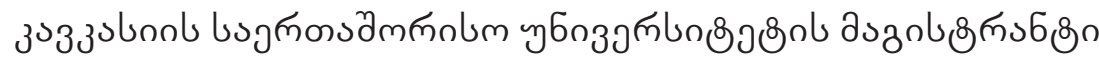
งm.ơmb\&s: elene.napo@gmail.com

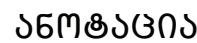

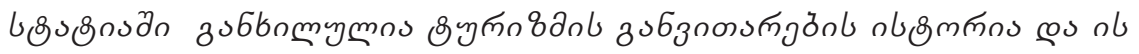

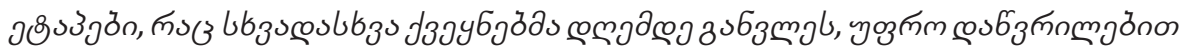

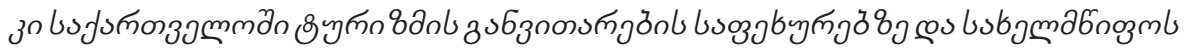

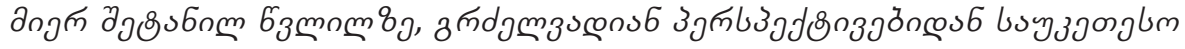

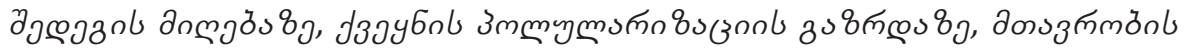

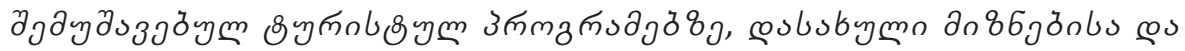

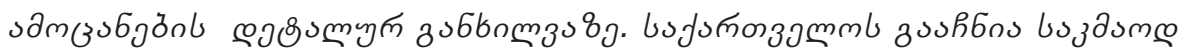

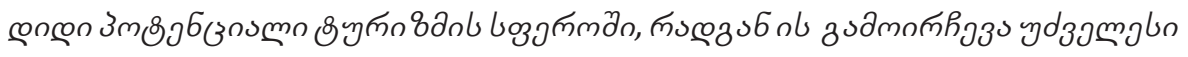

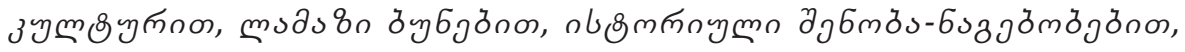

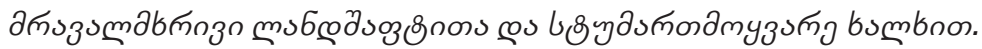

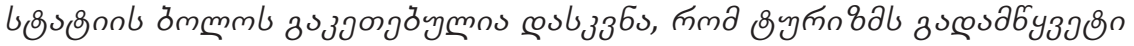

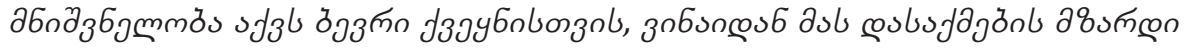

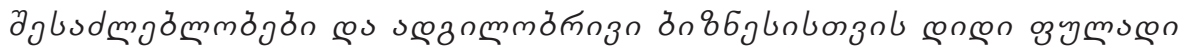

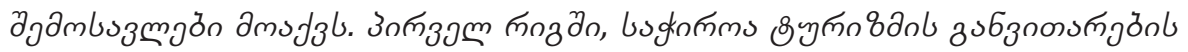

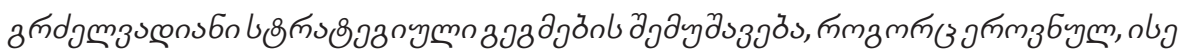

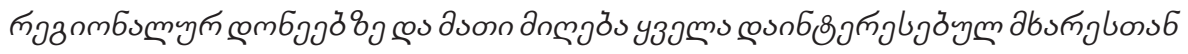

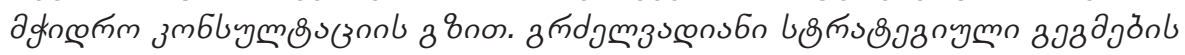

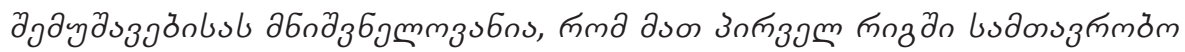

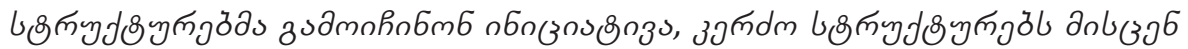

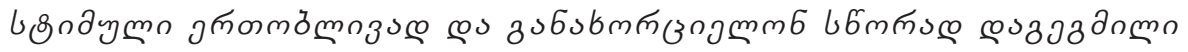

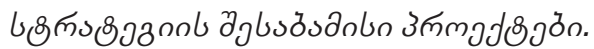

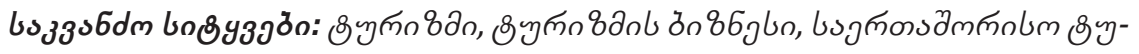

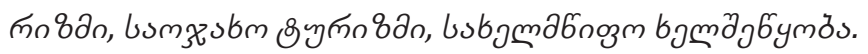




\section{วอเ১ว১m}

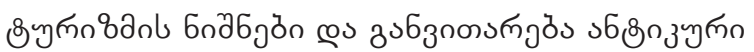

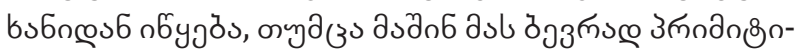

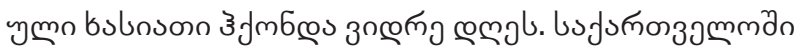

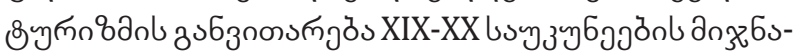

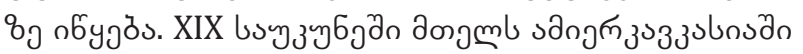

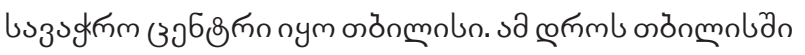

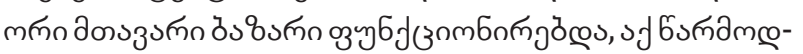

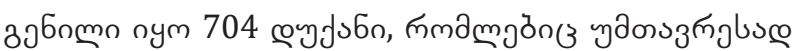

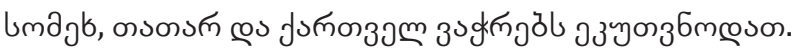

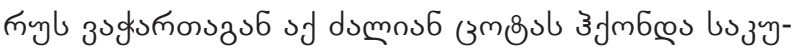

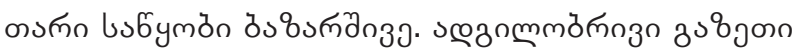

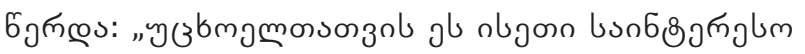

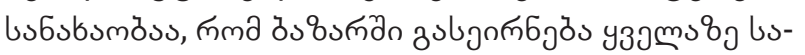

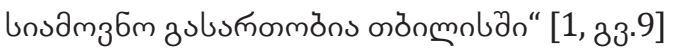

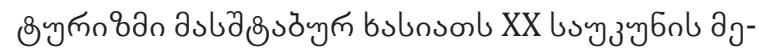

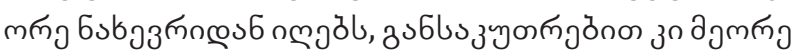

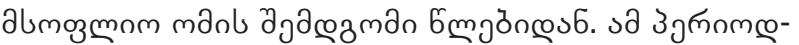

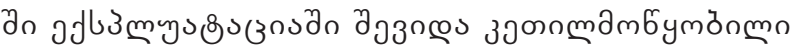

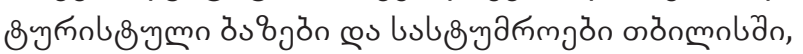

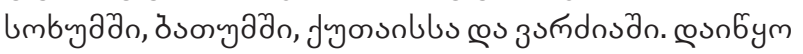

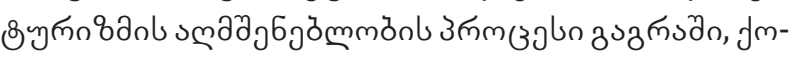

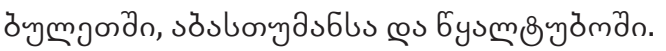

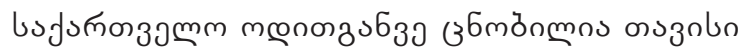

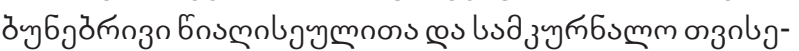

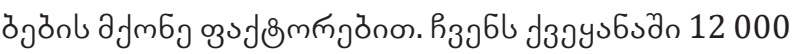

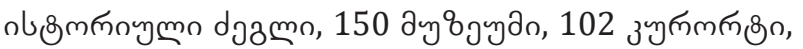
182 bs зулпмп

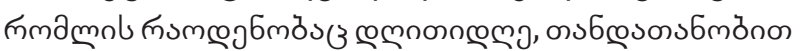

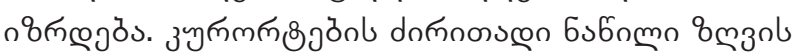

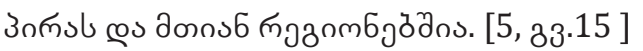

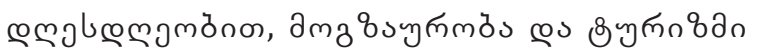

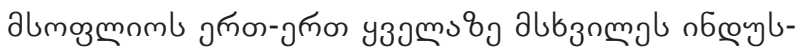

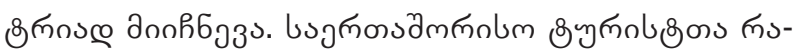

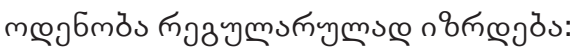

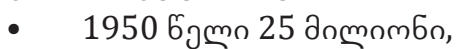

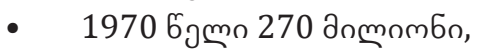

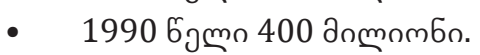

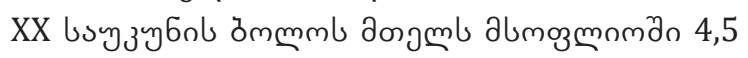

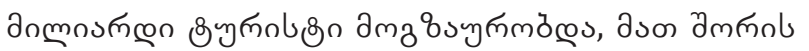

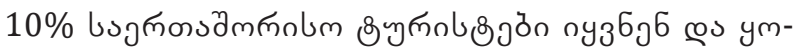

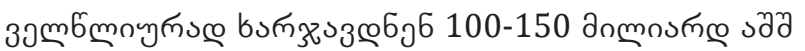

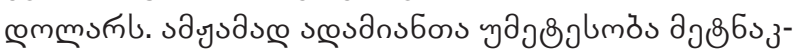

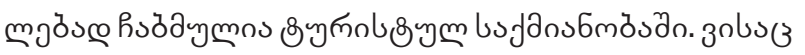

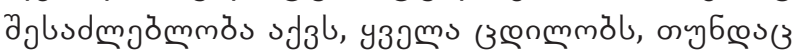

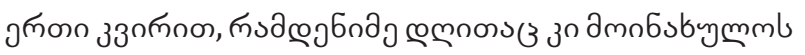

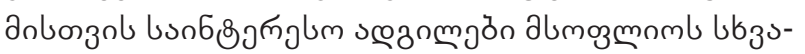

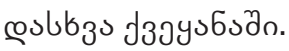

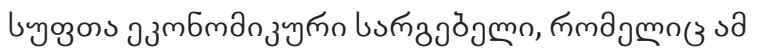

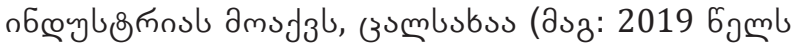

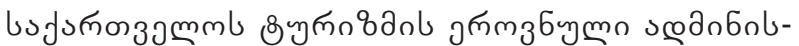

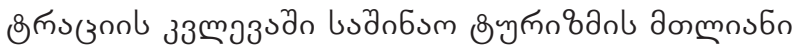

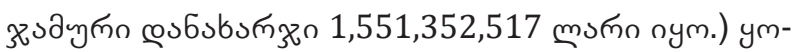

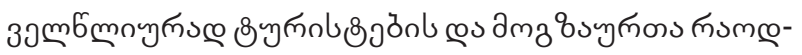

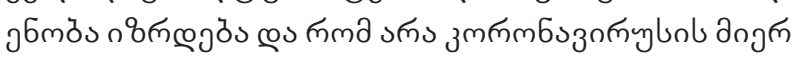

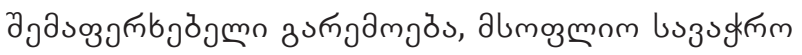

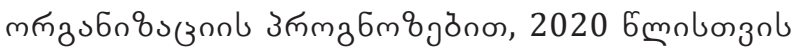

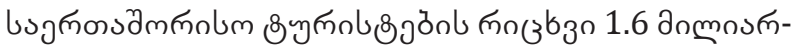

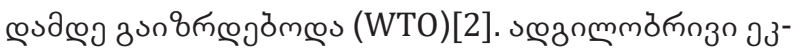

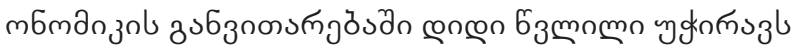

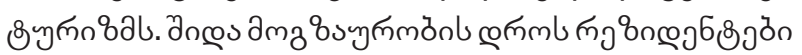

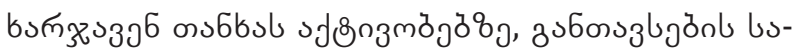

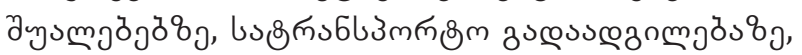

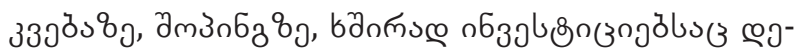

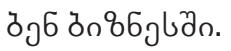

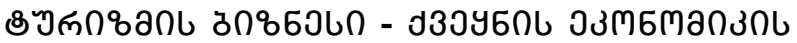

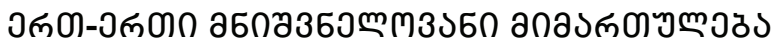

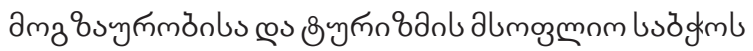

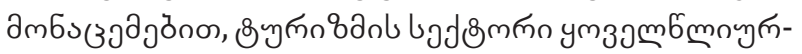

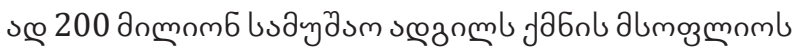
a

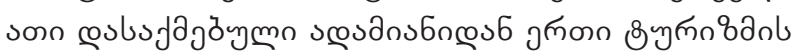

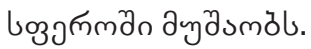

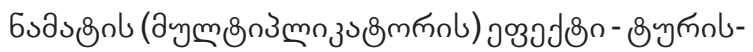

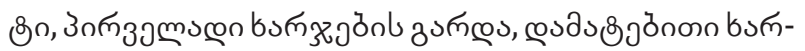

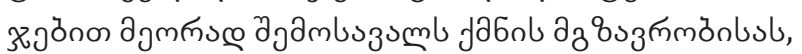
m

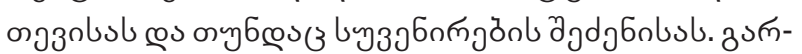

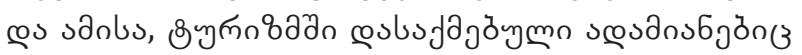

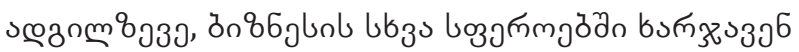

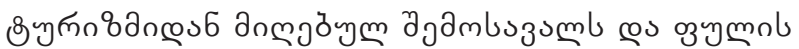

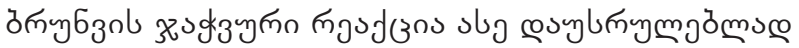

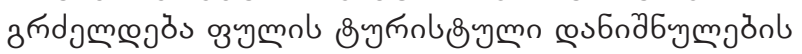

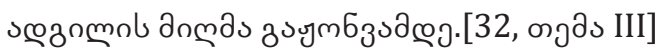

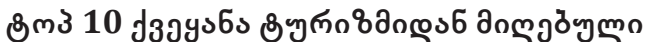

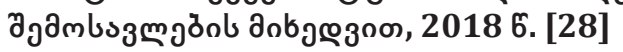

10. hกбวตก

9. nงउmbns

8. วงตว

7. s孔l 8 ñmos

6. n\&ेगmos

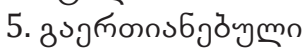

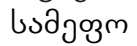

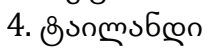

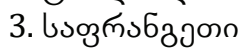

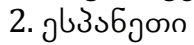

1. ১ว๖

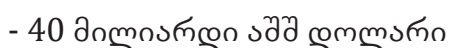

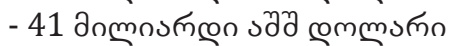

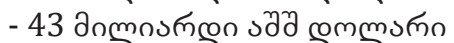

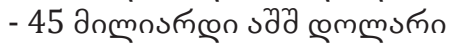

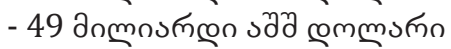

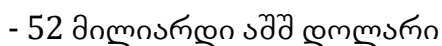

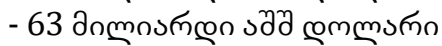

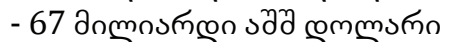

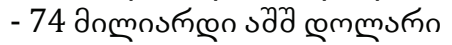

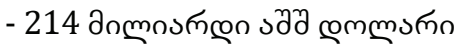




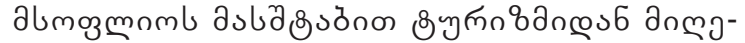

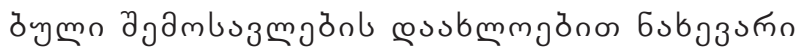

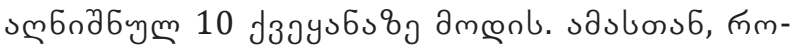

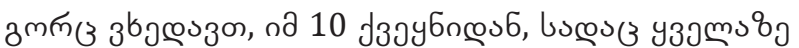

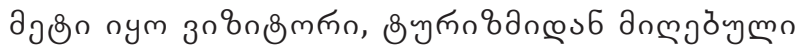

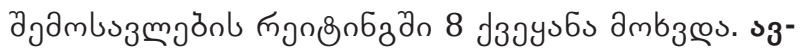

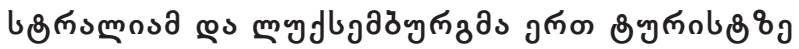

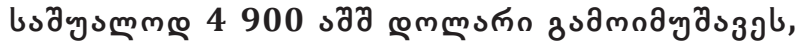

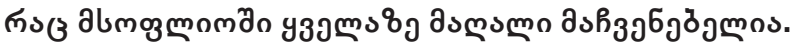

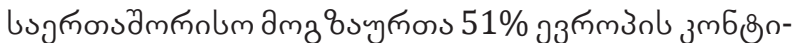

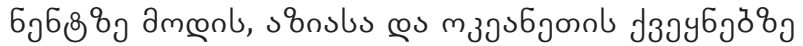

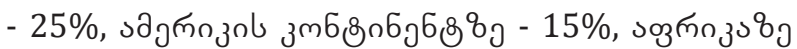

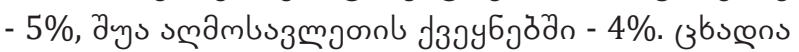

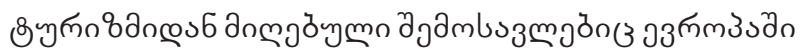
y3jms \&

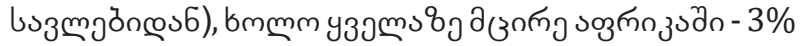

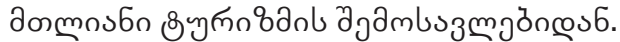

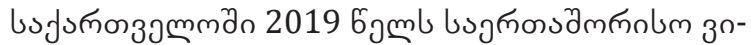

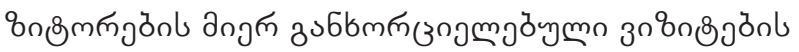

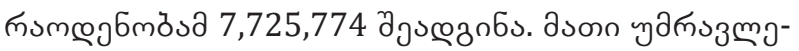

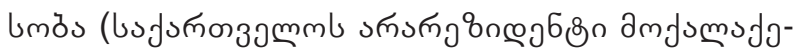

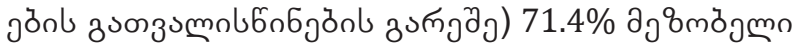

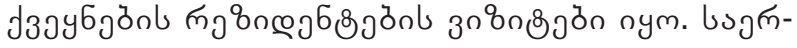

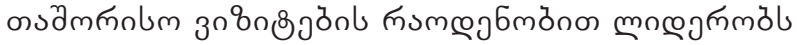

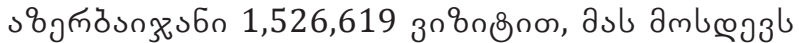

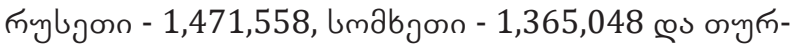

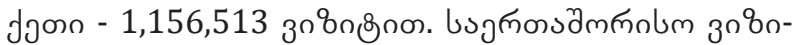

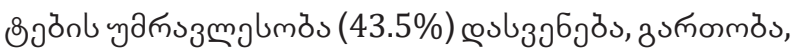

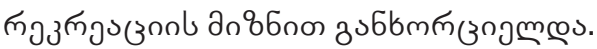

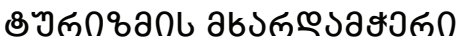

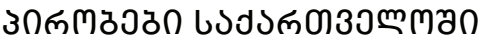

bus

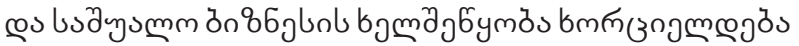

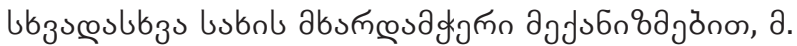

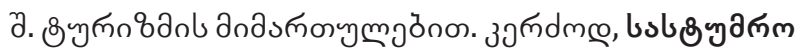

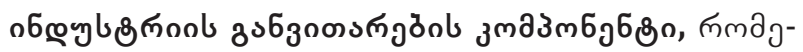

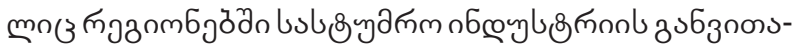

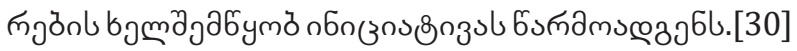

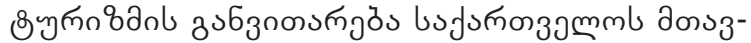

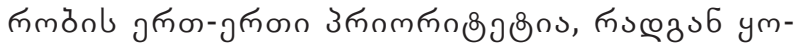

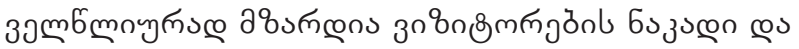

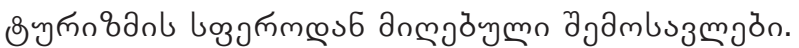

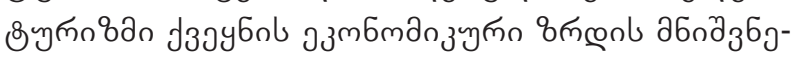

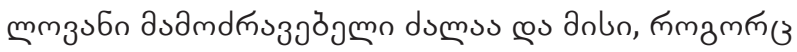

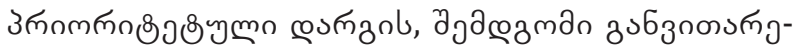

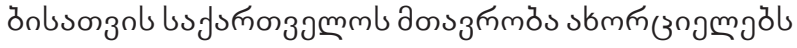

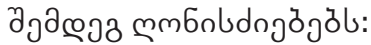

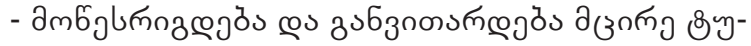

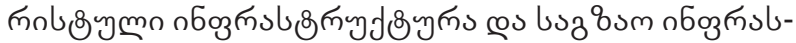

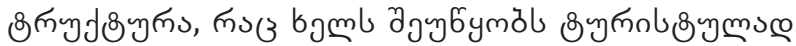

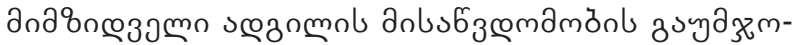

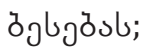

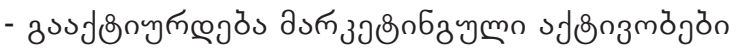

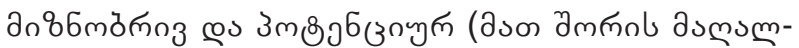

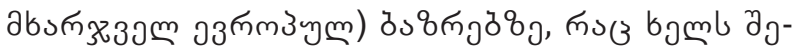

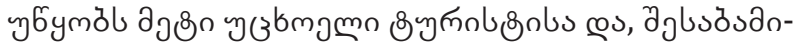

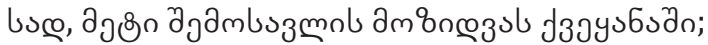

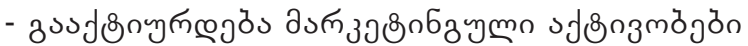

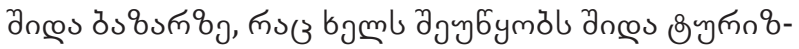

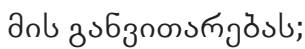

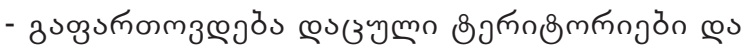

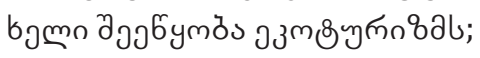

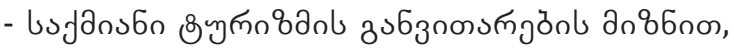

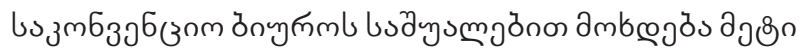

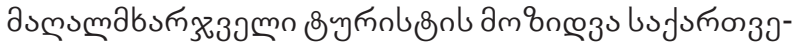

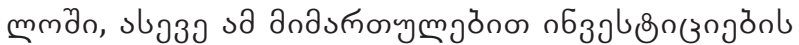

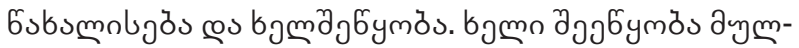

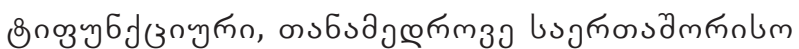

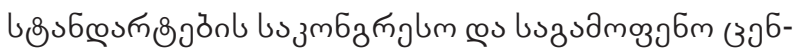

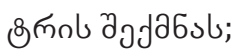

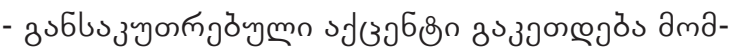

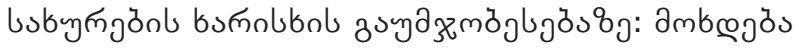

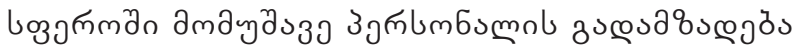

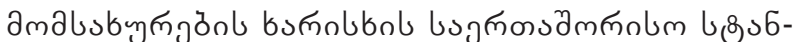

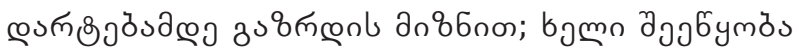

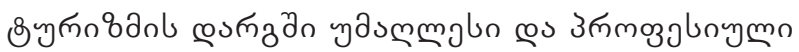

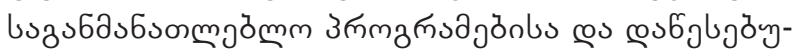

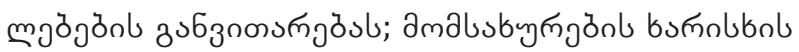

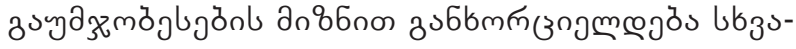

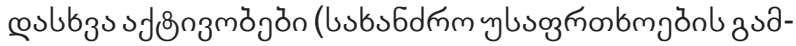

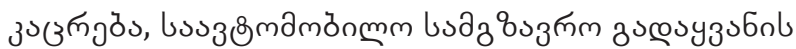

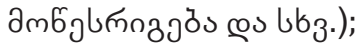

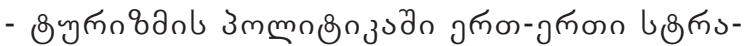

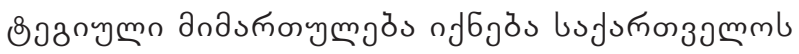

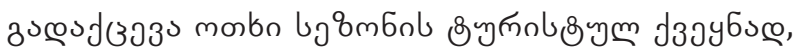

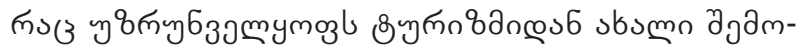

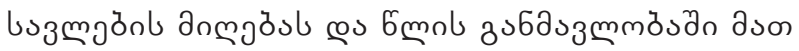

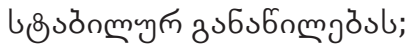

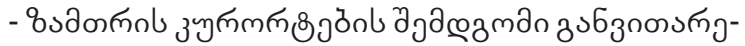

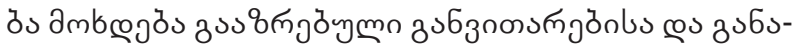

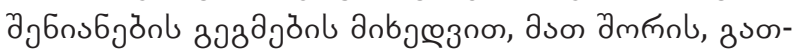

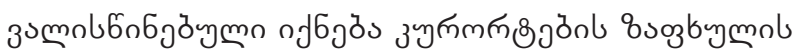

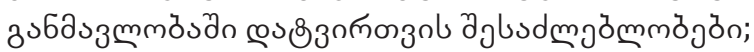

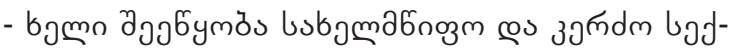

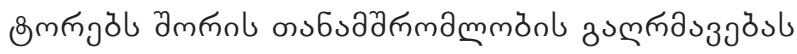

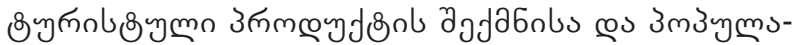
mo 


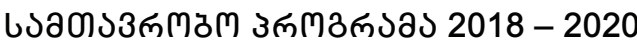

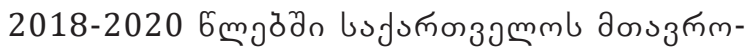

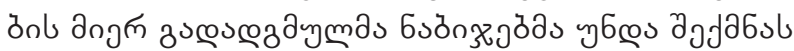

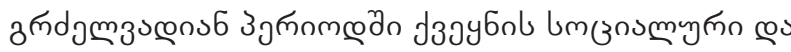

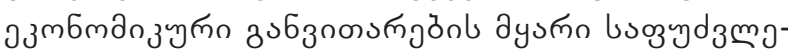

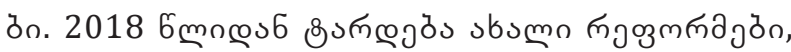

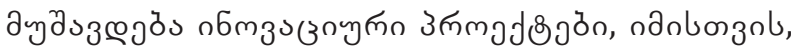

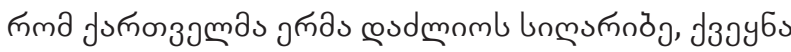

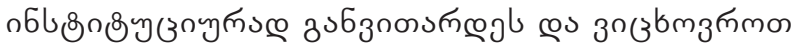

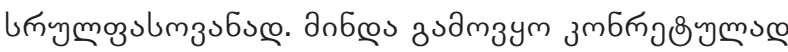

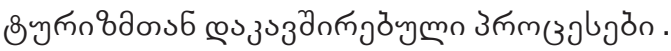

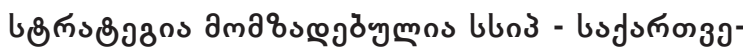

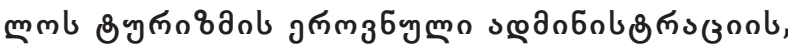

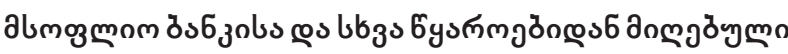

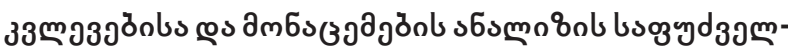
8. [24]

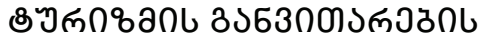

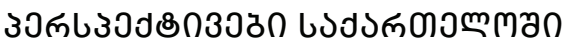

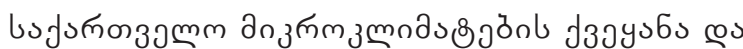
an sа১boss6, ononm Jy

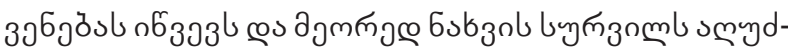

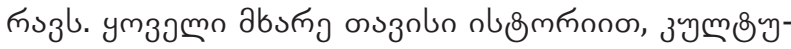

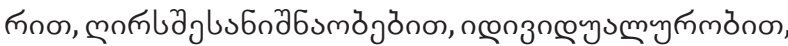

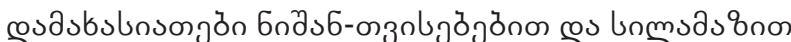

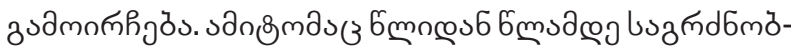

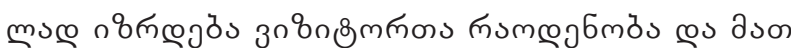

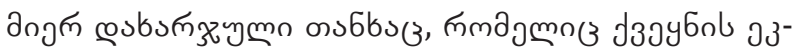
mbmanzul sdmngñal.

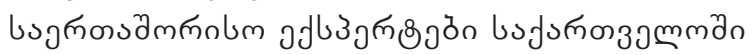

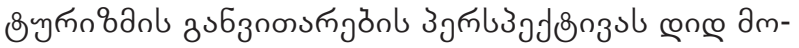

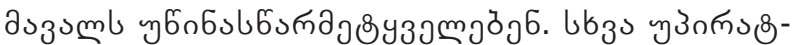

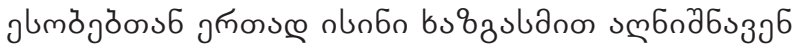

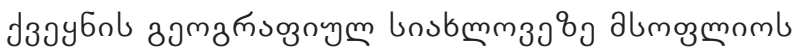

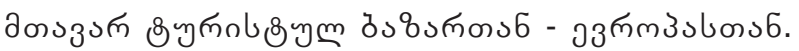

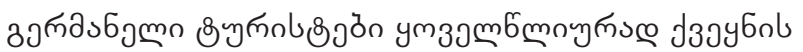

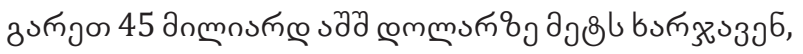

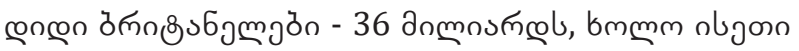

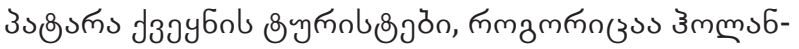

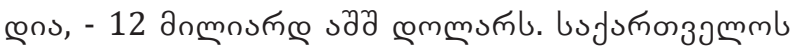

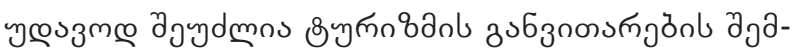

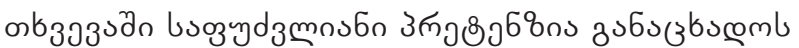

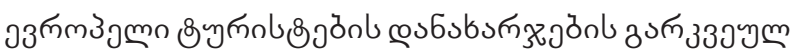

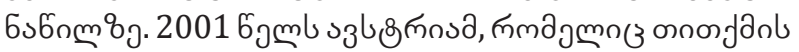

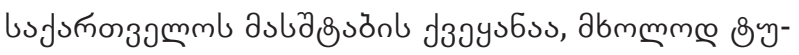

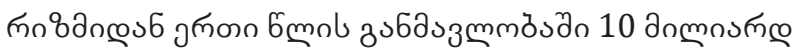

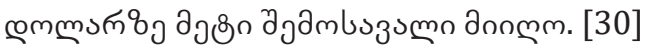

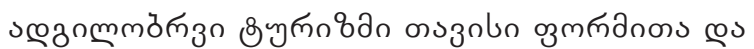

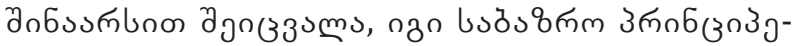

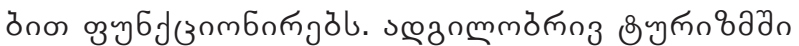

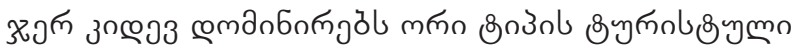

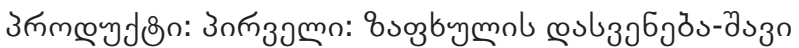

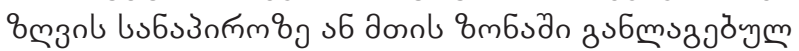

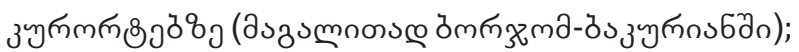

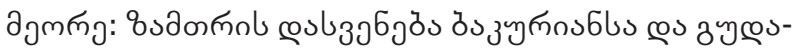

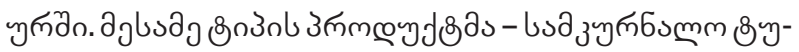

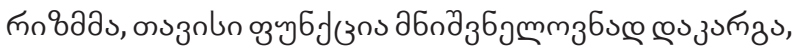

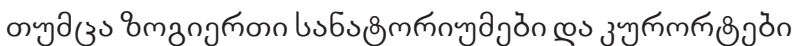

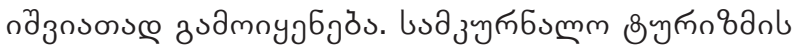

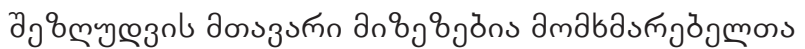

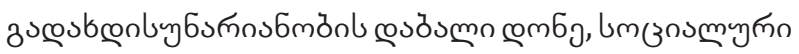

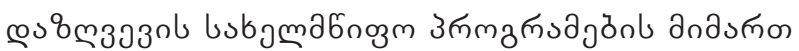

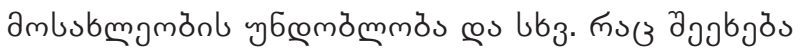

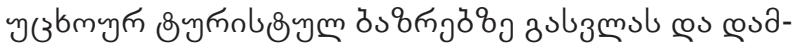

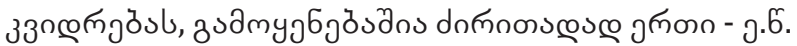

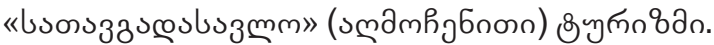

\section{œ১৬336১}

33м03

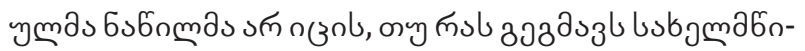

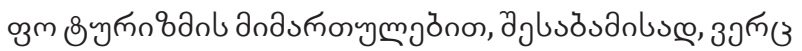

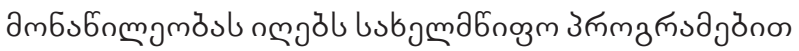

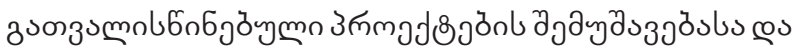

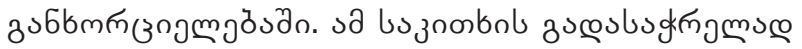

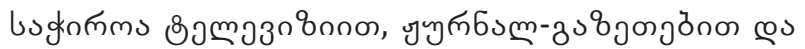

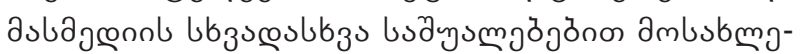

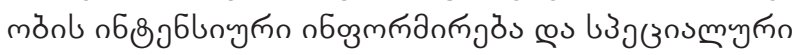

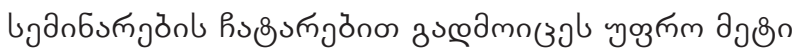

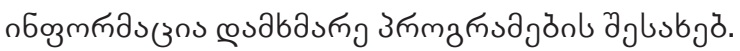

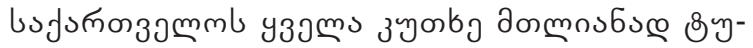

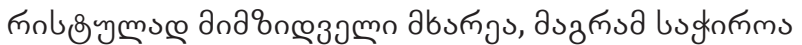

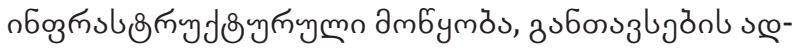

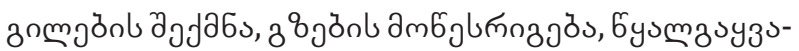

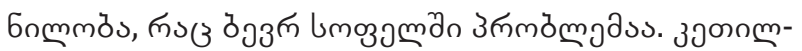

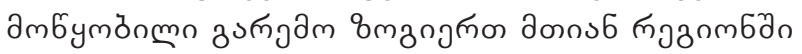

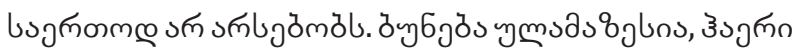

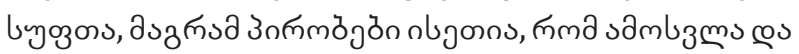

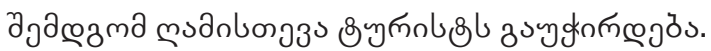

h h sஙn

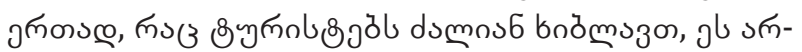

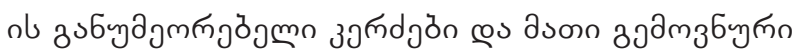

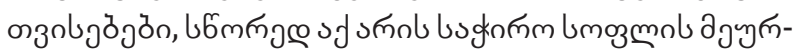

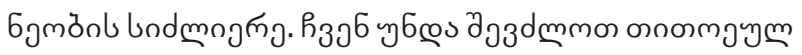

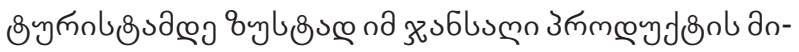

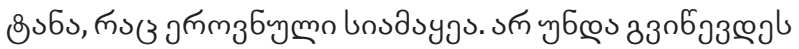

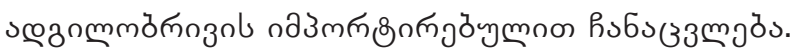




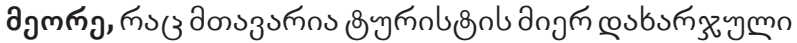

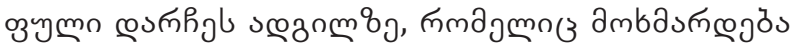

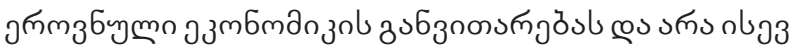

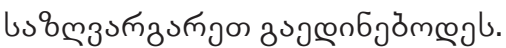

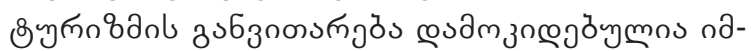

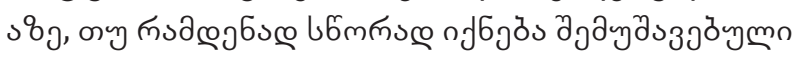

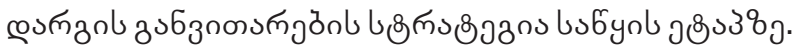

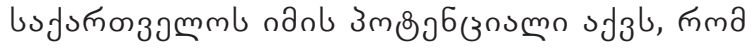

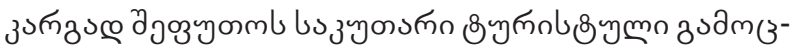

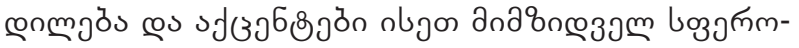

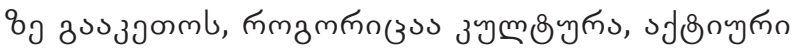

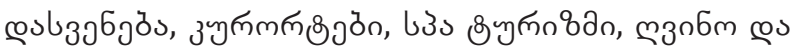

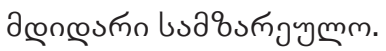

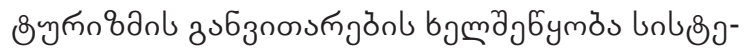

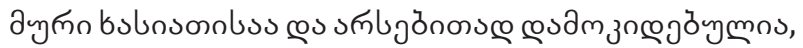

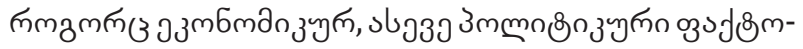

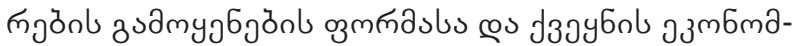

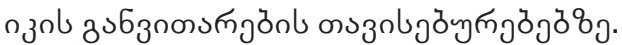

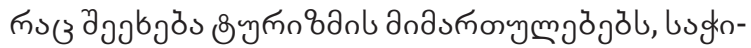

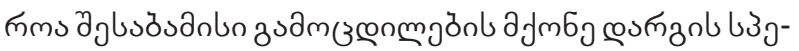

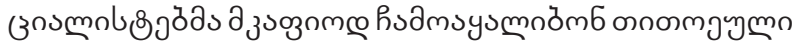

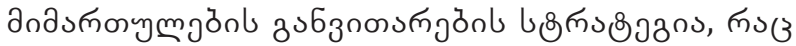

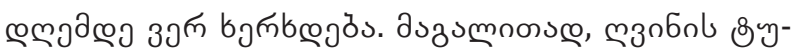

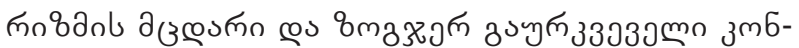

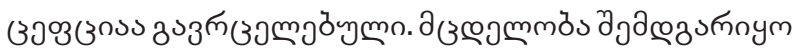

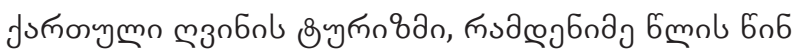

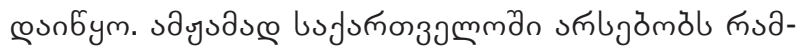

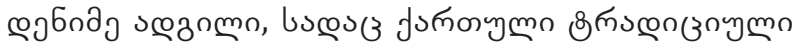

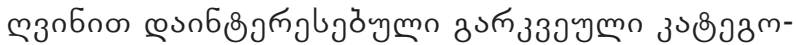

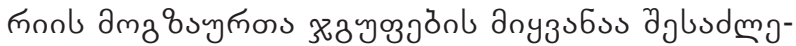

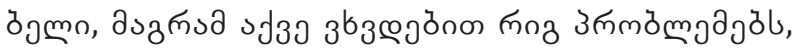

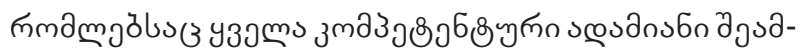

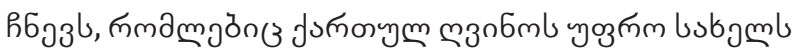

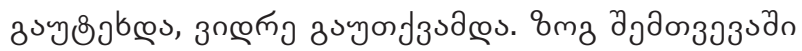

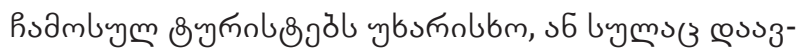

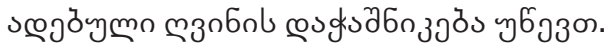

ง

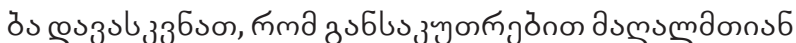

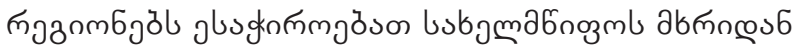

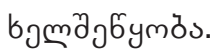

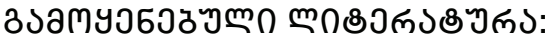

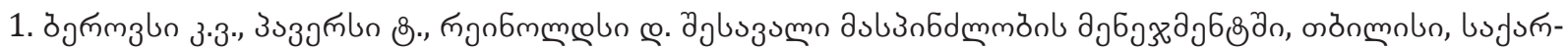

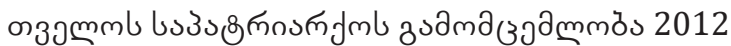

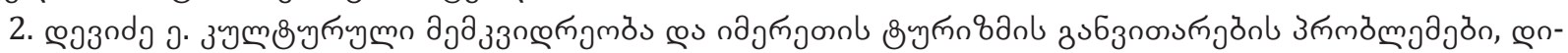

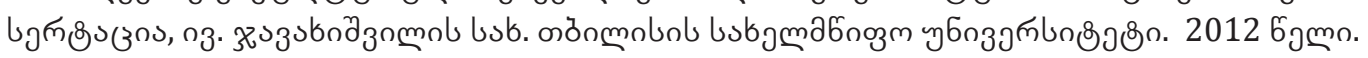

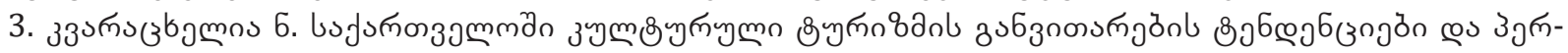

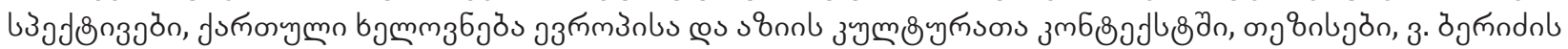

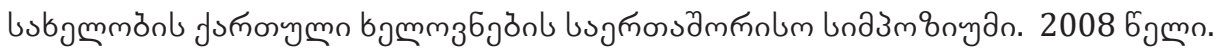

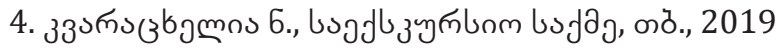

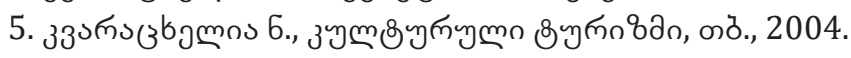

6. Karchava, L., 2012. Business-communications. Tbilisi, Loi, 328.

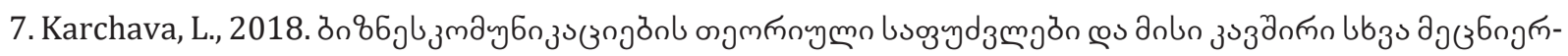

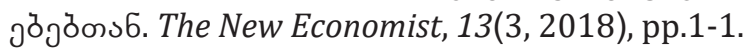

8. Kikutadze, V. and Tabatadze, L., 2016. Diversification of funding models of higher education service market in Georgia. In 5th EURASIAN MULTIDISCIPLINARY FORUM, EMF 2016 27-28 October, Tbilisi, Georgia (p. 56).

9. Lomia, E., 2020. The Evaluation of Russia's foreign policy towards Georgia following the 'Rose Revolution'. Journal of Liberty and International affairs, 6(1), pp.112-128.

10. Lomia, E., 2017. The United States-Russia Relations Before and After Russia's Intervention in Georgia and Ukraine.

11. LOMIA, E., Key Features of the US-Georgia Relations: Mutual Alliance and Strategic Partnership. SECTION II: Literature, Women's Issues, Education, p.54.

12. Lomia, T. and Lomia, E., 2020. ECONOMIC AND POLITICAL SUPPORT OF THE EUROPEAN UNION TO GEORGIA: RETROSPECTIVE ANALYSIS OF THE EU-GEORGIA RELATIONS. International Journal Vallis Aurea, 6(1), pp.35-43.

13. Lomia, T., 2018. The issue of forming rational economic policy of environmental protection. Globalization \& Business.

14. Lomia, T., 2015. The role of international environmental protection policy in Georgia. In Materials of reports made at the international scientific-practical conference held at Paata Gugushvili Institute of Economics 
of Ivane Javakhishvili Tbilisi State University in 2015 (p. 513).

15. Lordkipanidze R. E-Commerce Management (Handbook: Principles, World Statistics, Specifics of Georgia's Economic Priorities). - Tbilisi, 2020: 205.

16. http://dx.doi.org/10.13140/RG.2.2.26131.30244

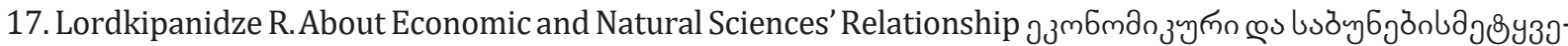

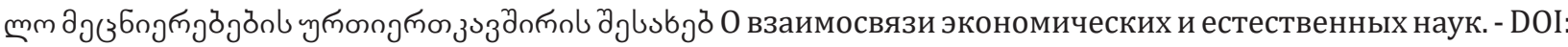
10.13140/RG.2.2.16952.08960, Tbilisi, The Public Academy named after Economist A. Gunia: 2020, Jan 7: 583.

18. http://dx.doi.org/10.13140/RG.2.2.16952.08960

19. Lordkipanidze R. Economic Outcomes of Smart Nutrition, Sports and Tourism E-Taxes

20. http://dx.doi.org/10.13140/RG.2.2.17169.25447

21. Pavliashvili, S., 2014. SUSTAINABLE DEVELOPMENT OF AGRICULTURE AND FOOD SECURITY IN GEORGIA. The Caucasus \& Globalization, 8(3-4), pp.55-61.

22. Pavliashvili, S., 2011. THE WORLD ECONOMIC CRISIS AND GEORGIA. The Caucasus \& Globalization, 5(34), pp.54-62.

23. Pavliashvili, S. and Gubeladze, D., AGRARIAN RURAL AND REGIONAL POLITICAL STRATEGIES IN AGRICULTURE. In Materials of reports made at the international scientific-practical conference held at Paata Gugushvili Institute of Economics of Ivane Javakhishvili Tbilisi State University in 2015 (p. 397).

24. Pavliashvili, S., PECULIARITIES OF STATE PROPERTY PRIVATIZATION AND ITS SOCIAL-ECONOMIC RESULTS IN GEORGIA. In Materials of reports made at the international scientific-practical conference held at Paata Gugushvili Institute of Economics of Ivane Javakhishvili Tbilisi State University in 2013 (p. 53).

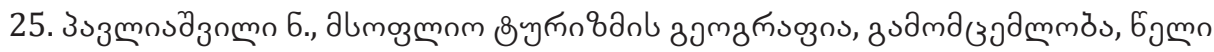

26. Tabatadze, L., 2015. Strategic directions of strengthening competition on higher education market of Georgia. European Scientific Journal.

27. Kikutadze, V. and Tabatadze, L., 2016. Diversification of funding models of higher education service market in Georgia. In 5th EURASIAN MULTIDISCIPLINARY FORUM, EMF 2016 27-28 October, Tbilisi, Georgia (p. 56).

28. Tabatadze, L., 2020. The New Vision of Modern Management Theory. The New Economist, 15(1, 2020), pp.1-1.

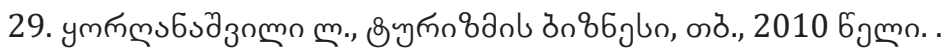

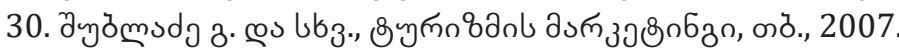

31. Boniface B., Cooper Ch. Worldwide Destinations. The Geography of Travel and Tourism. IV Edition. 2005 6əmo.

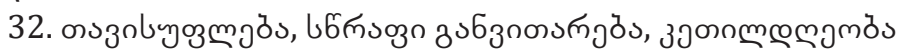

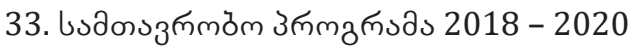

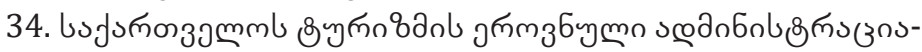

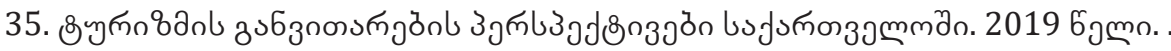

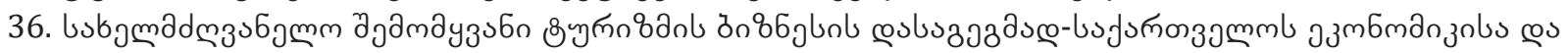

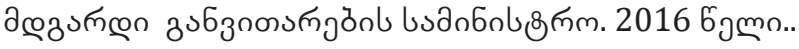

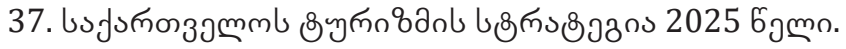

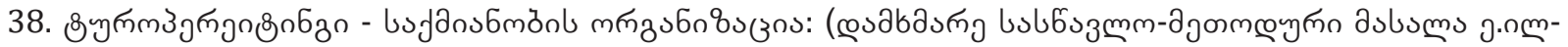

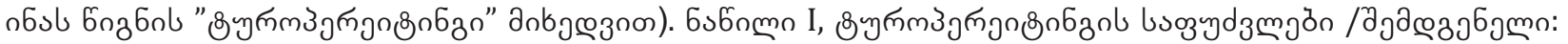

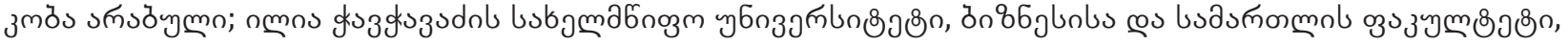

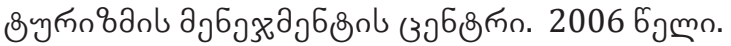

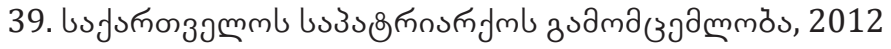

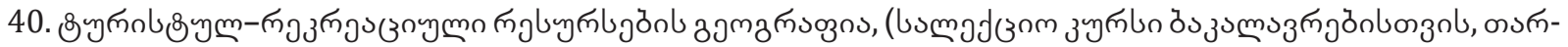

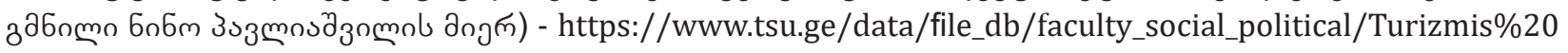
geografia-nino\%20pavliashvili.pdf

41. http://www.economy.ge/ \&̈ynn\&an

42. https://reginfo.ge/economic/

43. https://matsne.gov.ge/

44. https://tsu-tdi.blogspot.com/

45. http://press.tsu.ge/ 


\title{
TOURISM AS ONE OF THE PRIORITY DIRECTIONS IN GEORGIA
}

Loid Karchava

Doctor of Business Administration,

Professor.

Elene Kutaladze

Master of Caucasus International University

\begin{abstract}
The article discusses the history of the development and the stages that different countries have gone through so far, in details about the stages of the tourism development in Georgia. And about the contribution of the state, to the best results from the long term perspectives. It also focuses on the increase of the country's popularization, to the tourism programs developed by the government and for the detailed discussion of the set goals and objectives. Georgia has a great potential in the field of tourism, as it is distinguished by the ancient culture, beautiful nature, historic buildings, stunning landscapes and hospitable people.

The final part of the article concludes that tourism is crucial for many countries, as it brings growing employment opportunities and large monetary incomes for local businesses. First of all, it is necessary to develop long-term strategic plans for tourism development, both at the national and regional levels and adoption of them should be done through close consultation with interested sides. When developing long-term strategic plans, it is essential that the governmental structures take the initiative, give private incentives to private structures, and implement relevant projects of the well-planned strategies.
\end{abstract}

Keywords: tourism, tourism business, international tourism, family tourism, state support. 